\title{
Iron-borosilicate soft magnetic composites: the correlation between processing parameters and magnetic properties for high frequency applications
}

\author{
T. Gheiratmand, H. R. Madaah Hosseini, S.M. Seyed Reihani \\ Department of Materials Science and Engineering, Sharif University of Technology, Tehran, \\ 1458889694, Iran \\ *Corresponding author: Madaah@sharif.edu, t.gheiratmand@yahoo.com
}

\begin{abstract}
Iron-borosilicate soft magnetic composites are suitable magnetic materials for high temperature and high frequency applications. In this research two different techniques have been applied to fabricate these composites: uniaxial pressing following by sintering and spark plasma sintering. Different processing parameters including the content of borosilicate, the amount of compaction pressure and the size of iron particles have been evaluated through the study of microstructure and magnetic properties. The microstructural observations showed that the borosilicate is distributed on the iron grain boundaries enhancing the resistivity and causing the loss of eddy currents. Increasing the compaction pressure was led to the decrease of electrical resistivity. By increasing the frequency both real and imaginary parts of permeability decreased. The use of higher content of borosilicate resulted in the lower decreasing slop of permeability. Best combination of density, permeability and electrical resistivity was obtained in the sample with 2 wt\% of borosilicate. In addition, the densification of powders with fine particles was more difficult than coarse particles due to the inter particles friction and bridging effects. Furthermore, as the particles size increases the size of open porosities before sintering where the borosilicate could aggregate enhances. This could yields to the increase in the electrical resistivity. The high ratio of surface to the volume in the powders with fine particles results in the developing the demagnetizing fields and subsequently, decreasing the permeability. The highest relative density (99.99\% of theoretical density) with best distribution of borosilicate was achieved in the composites produced by spark plasma sintering (SPS). The relaxation frequency, obtained from imaginary part of permeability, was found as $340 \mathrm{~Hz}$ in the composites made by SPS.
\end{abstract}

Key words: Soft magnetic composite, Iron-borosilicate, Magnetic properties, Real and imaginary parts of permeability, Relaxation frequency 


\section{1- Introduction}

Recently, soft magnetic composites (SMC's) have been in the focus of interest due to their superior magnetic properties than silicone laminated steels and soft magnetic ferrites for application at high frequencies [1, 2]. These materials are comprised from iron particles insulated from each other by an organic coating which increase the electrical resistivity and decrease the losses relating to the eddy currents [2, 3]. SMC's could be applied in switch reluctance power supplies, DC output chokes, antilock braking sensors, resonant inductors, electromagnetic actuation devices, frequency filters, magnetic field shielding, transformers coil, and in high temperature applications such as electric components of aircraft engine [4].

The most common technique for production of SMC's is powder metallurgy. During this method the magnetic powder particles are surrounded by a dielectric insulating layer which causes barrier between particles for flow of eddy currents under AC applications [2, 4, 5]. However, the amount of insulating layer should be kept at the lowest level to have the optimum values for saturation magnetization and permeability [5].

In the recent years, different materials were utilized as a coating around iron powders such as phenolic resin [6, 7], Amorphous Vitroperm $\left(\mathrm{Fe}_{73} \mathrm{Cu}_{1} \mathrm{Nb}_{3} \mathrm{Si}_{16} \mathrm{~B}_{7}\right)$ alloy [8], silicone [9], phenolformaldehyde resin [10], $\mathrm{Al}_{2} \mathrm{O}_{3}$ [11], Parylenes [12], Mn-Zn ferrite [13], Ni-Zn ferrite [14-16], and $\mathrm{SiO}_{2}$ [17]. Although, the application of organic coatings lowers the processing temperature during warm compaction [2] it could also lowers the working temperature of composite. Moreover, the main problem accompanied with the addition of metallic compounds is the small electrical resistivity that is not too effective on avoiding the eddy current flow at high frequencies [8].

borosilicate glass, known as Pyrex, could be applied as an insulating material which raises the working temperature of soft magnetic composite. Actually, borosilicate is a type of glass with silica and boron trioxide as the main glass-forming constituents. The softening point of this glass (the temperature at which the viscosity is approximately 107.6 poises) is $\sim 820^{\circ} \mathrm{C}$. Thus, it is an appropriate candidate for insulating the iron particles and subsequently, decreasing the eddy currents at high temperature and high frequency applications.

To fabricate the soft magnetic composites different methods have been applied so far which are on the base of powder compaction following by sintering or annealing treatments. Designing the die which could withstand either the high pressures (in the order of GPa) or high temperatures is 
the main concern of the conventional powder techniques. Obtaining the fully dense sample is another difficulty of these processes [8].

Spark plasma sintering (SPS) is a unique powder metallurgy technique in which the dense and net shape samples could be produced. In this process a DC on-off pulse is created which passes through the graphite die as well as the sample enabling the material to be heated homogenously and simultaneously from both inside and outside. The short heating time of about $10 \mathrm{~min}$ outstands the SPS among the conventional sintering processes [18].

In this research, the fully dense iron- borosilicate soft magnetic composite has been fabricated by spark plasma sintering technique. Furthermore, the effect of borosilicate content and iron particles size has been studied through the evaluation of microstructure and investigation of high frequency magnetic properties.

\section{2- Experimental procedure}

Iron powders were sieved and separated into the three groups with particles size of $\mathrm{d}<45$, $45<\mathrm{d}<125$ and $\mathrm{d}>125 \mu \mathrm{m}$. The purity of iron powders was above $99.5 \%$ containing $0.02 \% \mathrm{C}$, 0.05\% Si, $0.015 \%$ P, $0.015 \%$ S, 0.15\% Mn. Borosilicate powder was obtained by milling of Pyrex glass, powders smaller than $63 \mu \mathrm{m}$ were used. Mixing of iron and borosilicate powders were accomplished in a Turbula mixer. To determine the effect of borosilicate content, three different amounts of 1, 2 and 3 wt\% were utilized. The powders were consolidated using two different techniques: uniaxial pressing following by sintering and spark plasma sintering. Uniaxial pressing was conducted at high pressures in the order of GPa using a special die designed from tool steel. The sintering of samples was carried out at $\sim 800{ }^{\circ} \mathrm{C}$. At first, the optimum content of borosilicate was determined, and then the effect of particles size on the magnetic properties was investigated.

To get the full dense sample, the compaction of powders was carried out by means of a SPS equipment (model: Nanozint10) at temperature of $\sim 800{ }^{\circ} \mathrm{C}$. The powders were charged in a graphite die with the inner diameter of $25 \mathrm{~mm}$ lined by a graphite paper. Afterwards, the die was inserted in a vacuum chamber evacuated up to $6 \times 10^{-2}$ Torr at room temperature. The pressure of compaction was $40 \mathrm{MPa}$.

Consolidated alloys were sanded and polished in order to remove the graphite paper and carburized layer from the surface. The density of bulk samples was measured by the 
Archimedean method. The microstructure of sintered samples was studied using a Field Emission Scanning Electron Microscope, FESEM (MIRA3-TESCAN). The electrical resistivity of samples was determined by means of 4 point probe test. Complex permeability consisting of real and imaginary parts were evaluated using an inductance/capacitance/resistance meter, LCR meter (8110G, Instech). It should be mentioned that the inductance and AC resistance of the samples were measured to characterize the magnetic permeability. Real and imaginary parts of relative permeability were calculated using the following relationships:

$$
\begin{aligned}
& \mu^{\prime}=\frac{L_{s}}{L_{0}}=\frac{L_{s} l_{e}}{\mu_{0} N^{2} A_{e}} \\
& \mu^{\prime \prime}=\frac{R_{a c}}{\omega L_{0}}=\frac{R_{a c} l_{e}}{\omega \mu_{0} N^{2} A_{e}}
\end{aligned}
$$

where, $L_{s}$ and $L_{0}$ are the inductance of solenoid with and without core, $R_{a c}$ is the AC resistance of the core, $\omega$ is the angular frequency $(2 \pi f), N$ is the number of turns of the coil, $A_{e}$ is the area of cross-section and $\mathrm{l}_{\mathrm{e}}$ is the mean flux density path of the sample. It should be said that $\mathrm{L}_{0}$ could be calculated from the geometrical relations $[8,10]$.

The DC hysteresis loops were recorded at room temperature using a Vibrating Sample Magnetometer (VSM) device at external magnetic field up to $10 \mathrm{kOe.}$

\section{3- Results and discussion}

\section{3-1- The compaction by uniaxial pressing technique}

\section{3-1-1- The optimum content of borosilicate}

To study the effect of borosilicate content, the compaction was carried out at pressures of 800, 1100, $1300 \mathrm{MPa}$ for all three samples. Since the sample with $3 \mathrm{wt} \%$ of borosilicate could not fully compacted by the mentioned pressures, the additional pressures of 1500 and $1700 \mathrm{MPa}$ were also applied.

The weight percent, volume percent and theoretical density of composites with different borosilicate contents have been summarized in Table 1. It should be noted that the volume percent has been obtained using the weight percent value regarding the densities of iron and borosilicate as 7.8 and $2.23 \mathrm{~g} / \mathrm{cm}^{3}$, respectively. Moreover, the theoretical density has been calculated using the following relationship:

$$
\rho_{\text {th }}=\rho_{\text {Borosilicate }} \times \text { vol } \% \text { Borosilicate }+\rho_{F e} \times \text { vol\% } F e
$$


Table 1. The weight and volume percents of borosilicate together with the calculated theoretical density in the different composites

\begin{tabular}{c|c|c|c}
\hline Borosilicate weight percent & 1 & 2 & 3 \\
\hline Borosilicate volume percent & 3.41 & 6.66 & 9.76 \\
\hline Theoretical density $(\mathrm{g} / \mathrm{cm} 3)$ & 7.60 & 7.42 & 7.25 \\
\hline
\end{tabular}

Fig. 1 shows the relative density (the ratio of the measured density to the theoretical density) as a function of the compaction pressure in the samples with different contents of borosilicate. Evidently, the density decreases with increasing the insulating material. This is due to the more distributed air gaps develop in the sample. In addition, the relative density increases with enhancing the pressure. Actually, the main mechanism of increasing the density during the compaction with applied high pressures in the range of GPa is the plastic deformation of powder particles. Borosilicate particles are ceramic powders which could not plastically deform as iron powders. As could be seen in Fig. 1, at a constant pressure, e.g. $1300 \mathrm{MPa}$, the sample with higher amount of borosilicate ( $3 \mathrm{wt} \%$ ) has the lower relative density.

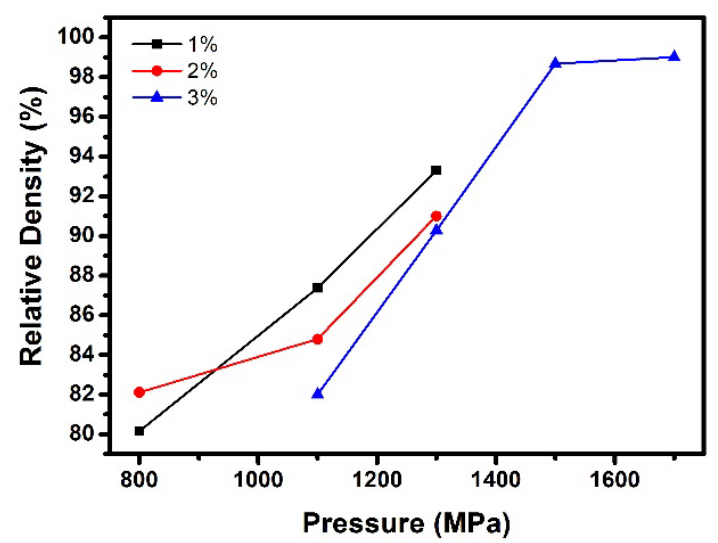

Fig.1. The density as a function of compaction pressure in the samples with different borosilicate contents.

Fig. 2 illustrates the secondary and back-scattered images of microstructure at two magnifications for composites containing different amounts of borosilicate compacted at 1300 MPa. As evident, by increasing the borosilicate content its distribution becomes more homogenous. In addition, more particles are coated by insulating layer. The borosilicate particles mostly situate on the grain boundaries and triple junctions. The EDX analysis revealed that the phases with light and dark color (A and B) are related to iron and borosilicate, respectively. The 
composition of these phases has been listed in Table 2. The secondary images also exhibit that by increasing the borosilicate content the amount of porosities enhances, the compaction becomes more difficult and thus the density decreases. It should be mentioned that the similar microstructures have been observed by the other researchers on iron powders coated by silicone [9] and epoxy resin [12].
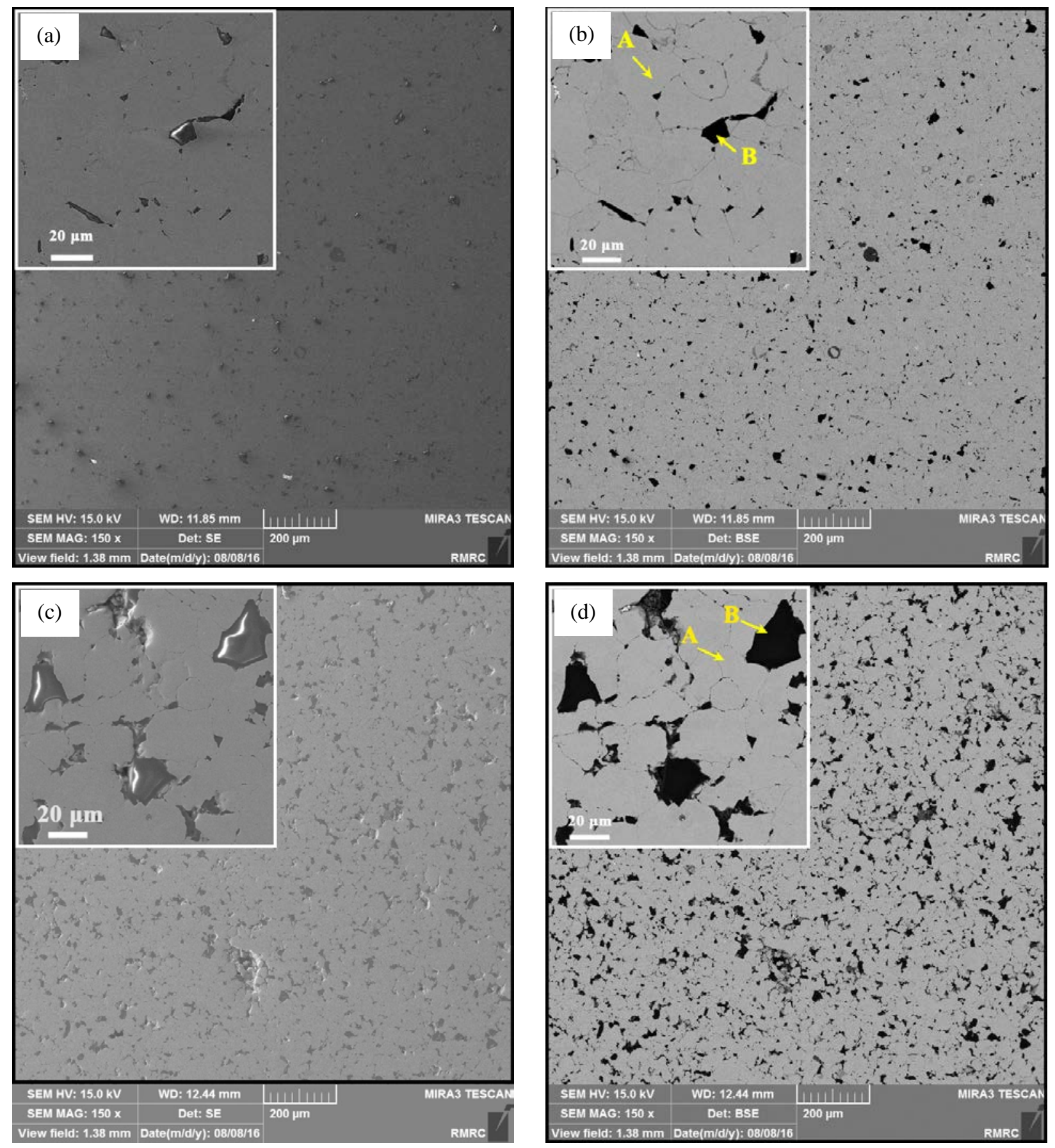

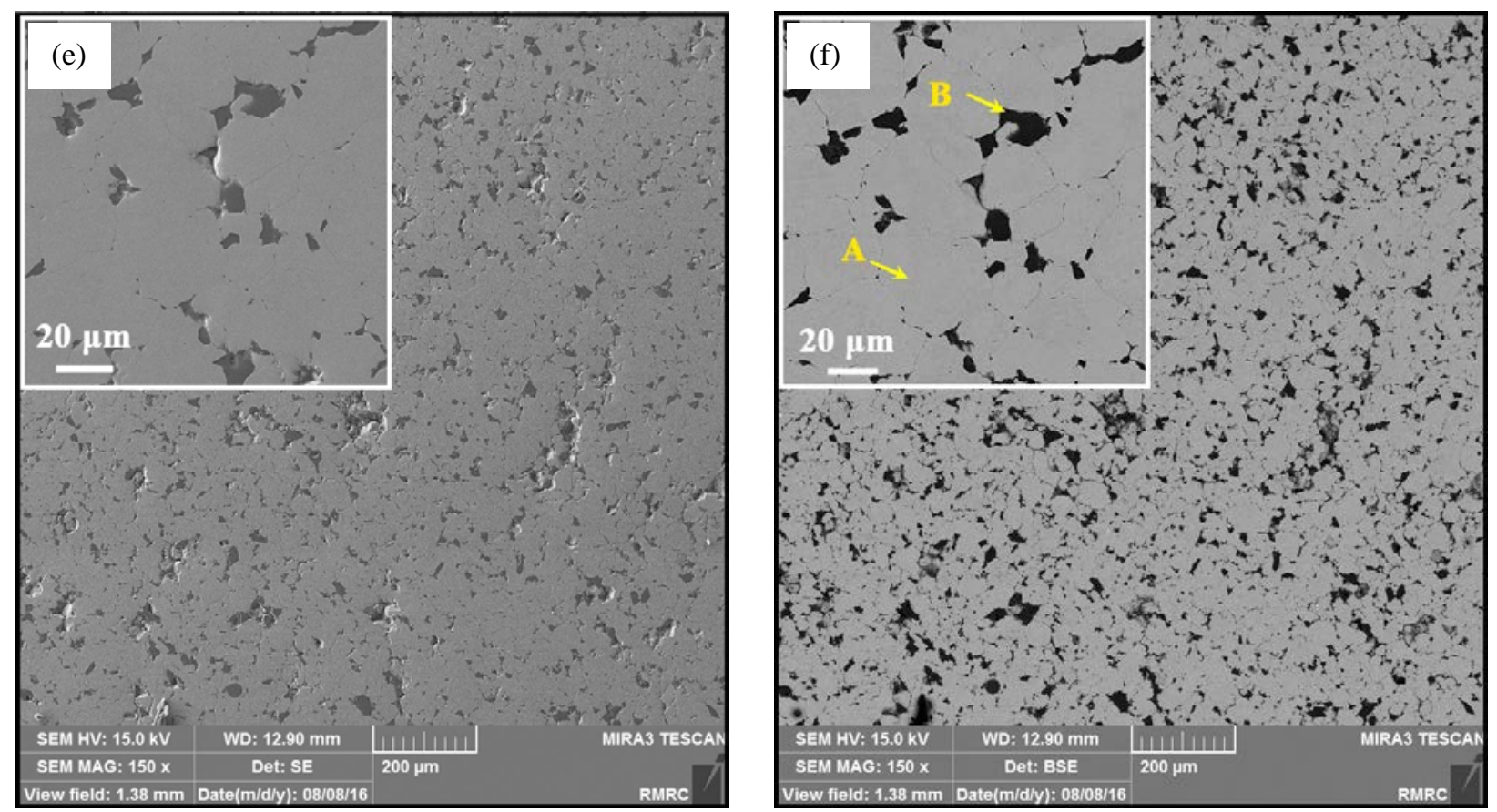

Fig.2. The secondary and back-scattered images of microstructure of composites containing different amounts of borosilicate, a,b) 1, c,d) 2 and e,f) 3 wt\%.

Table 2. EDX analysis of different phases shown in the SEM images on Fig.2

\begin{tabular}{c|c|c|c|c|c|c|c}
\hline $\begin{array}{c}\text { The percent of borosilicate } \\
\text { in Composite (wt\%) }\end{array}$ & $\begin{array}{c}\text { Elements } \\
\text { (atomic percent) }\end{array}$ & Fe & B & O & Na & Al & Si \\
\hline \multirow{2}{*}{1} & A & 100 & - & - & - & - & - \\
\cline { 2 - 9 } & B & - & 16.33 & 64.11 & 1.58 & 0.67 & 17.32 \\
\hline \multirow{2}{*}{2} & A & 100 & - & - & - & - & - \\
\cline { 2 - 9 } & B & - & 21.23 & 59.78 & 1.38 & 0.65 & 16.96 \\
\hline \multirow{2}{*}{3} & A & 100 & - & - & - & - & - \\
\hline & B & 12.77 & 21.97 & 48 & 0.39 & 9.64 & 7.24 \\
\hline
\end{tabular}

The changes of electrical resistance as a dependence of compaction pressure in samples with different amounts of borosilicate have been presented in Fig. 3. As explained before, increasing 
the compaction pressure results in the plastic deformation of iron particles and consequently, the elimination of porosities and gaps. The air gaps act as barrier for movement of electrons thus they increase the electrical resistance. Therefore, reducing the air gaps leads to the decrease of electrical resistance. With increase of borosilicate content, on one hand the thickness of insulating material around each particle enhances. On the other hand the coating of more particles takes place. Hence the increase of electrical resistivity is expected.

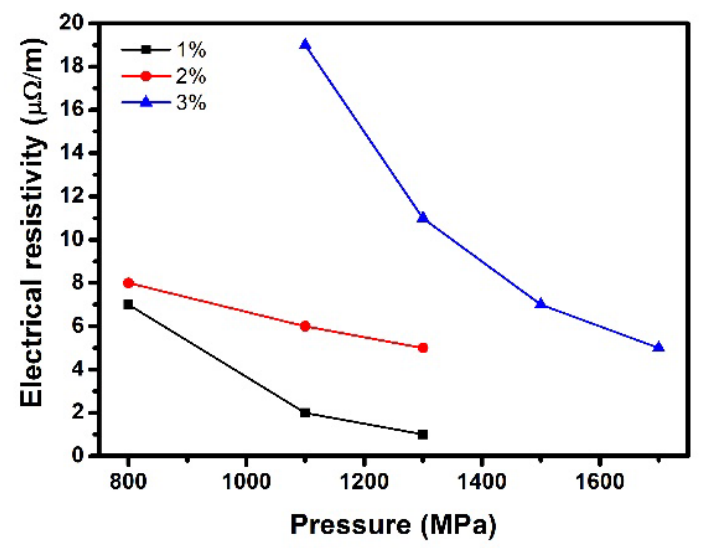

Fig.3. The electrical resistance as a function of compaction pressure in samples with different borosilicate contents.

The DC hysteresis loop of two composite samples containing $3 \mathrm{wt} \%$ of borosilicate with the highest density has been indicated in Fig.4. The magnetization of samples with density of 98.67 and $99 \%$ of theoretical density are 175 and 178 emu $\mathrm{g}^{-1}$, respectively. Furthermore, the coercivities of these samples are 12 and 10 Oe, respectively. Actually, with increasing the density the magnetization increases and coercivity reduces. The higher value of coercivity in the sample containing more porosity (lower density) is owing to the magnetostatic phenomenon. According to this phenomenon, an internal demagnetizing field resulting from the surface monopoles of porosities is generated. Thereby, the higher external field is needed to overcome the internal field, thus the coercivity increases. 


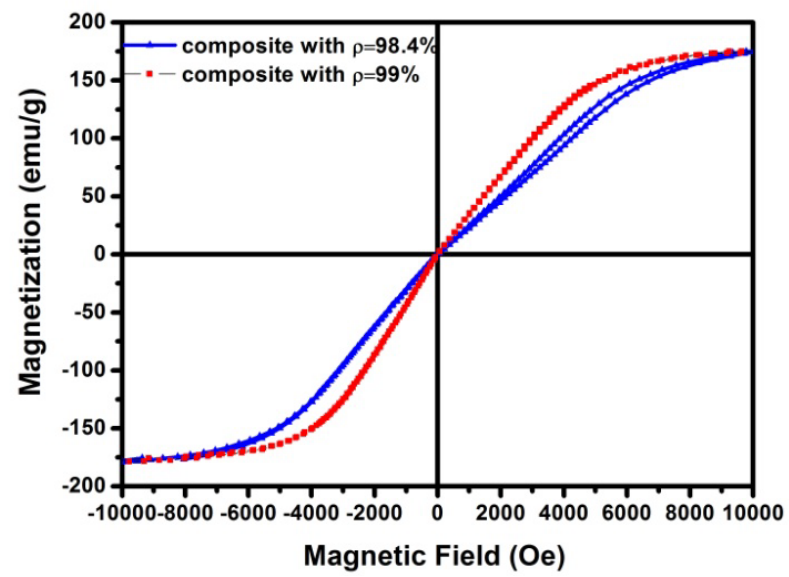

Fig.4. The DC hysteresis loop of samples with different densities.

The real and imaginary parts of relative permeability for composites with different contents of borosilicate have been exhibited in Fig.5. The real part of permeability ( $\left.\mu^{\prime}\right)$ strongly depends on the amount of non magnetic phases, density, number of porosities, crystal and magnetic anisotropies. The imaginary part of complex permeability reveals the losses relating to the eddy currents and hysteresis response [8]. Obviously, the value of both parts of relative permeability is higher in the sample with lower amount of non-magnetic borosilicate phase. The maximum value of real part for samples with 1, 2 and $3 \mathrm{wt} \%$ of borosilicate is 160, 90 and 60 , respectively. Furthermore, the maximum value of imaginary part of permeability for the mentioned samples is 16.7, 13.5 and 11.5, respectively. It should be noted that the value of real part of permeability is in line with what reported previously [12].
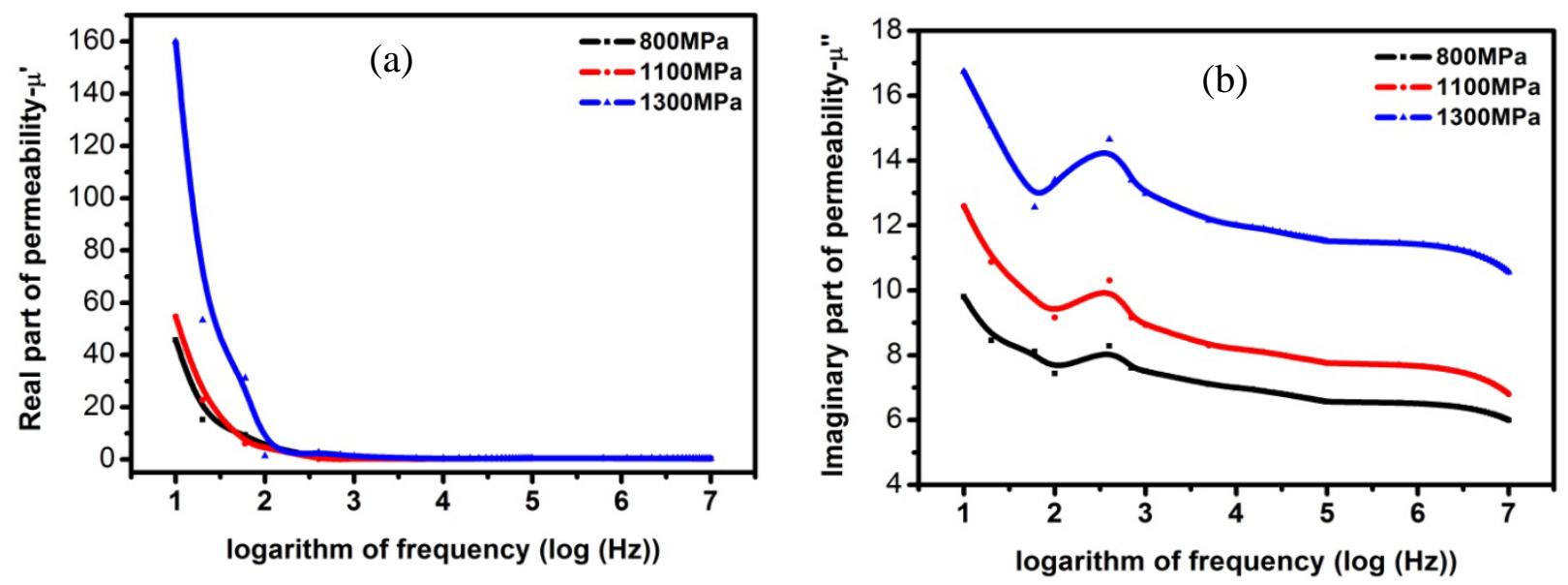

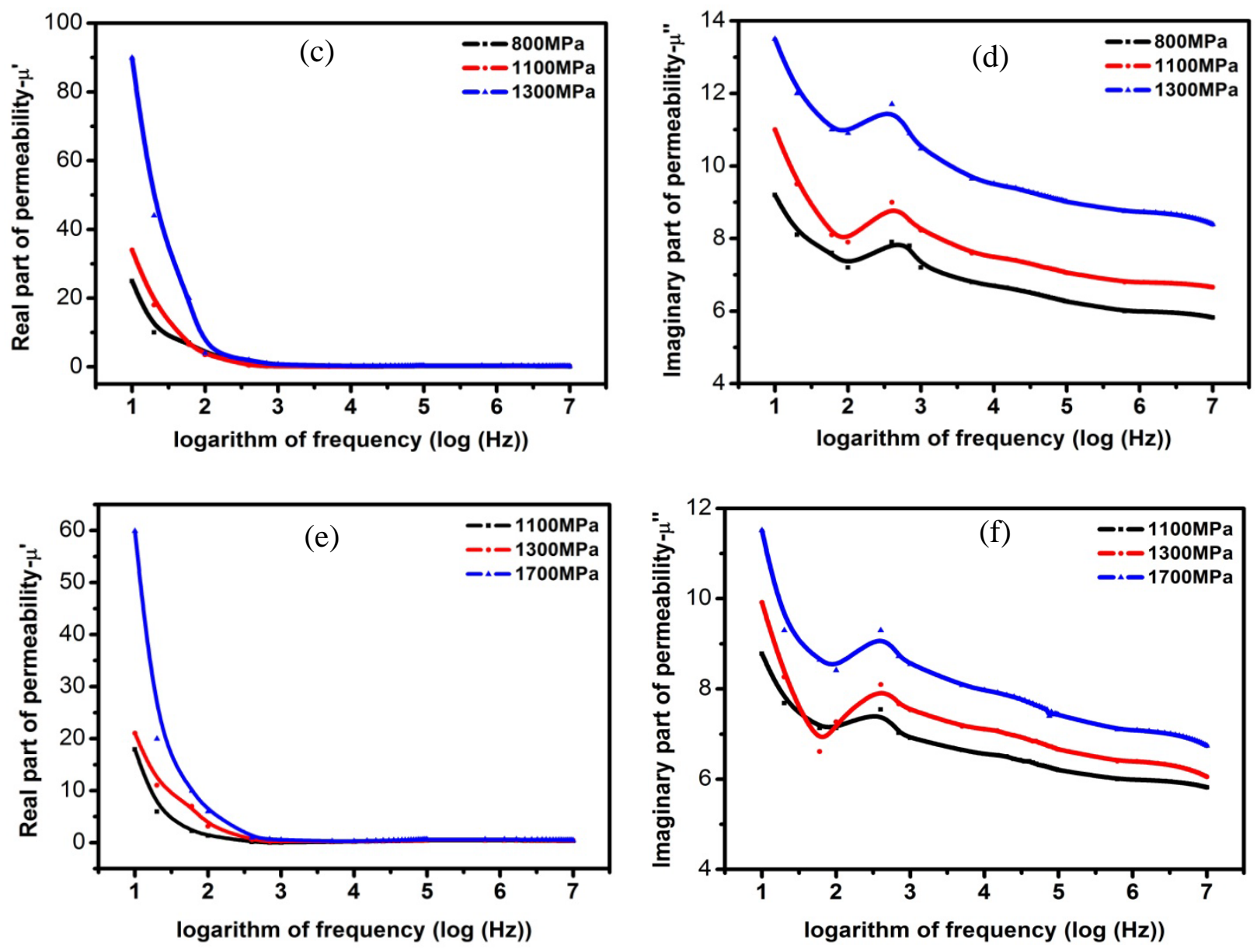

Fig.5. Real and imaginary parts of complex permeability for composites with different borosilicate contents of a,b)

$$
\text { 1, c,d) } 2 \text { and e,f) } 3 \text { wt } \% \text {. }
$$

As could be clearly seen, both parts of permeability decrease with increasing of frequency. The drop of permeability is significant at the initial frequencies below $\sim 320 \mathrm{~Hz}$, afterwards, it reduces slowly and smoothly. Moreover, it could be seen that the permeability is higher for samples with higher density. This is a result of eliminating porosities at higher compaction pressures and consequently, the existence of more magnetic mass at the same volume. This result is in line with what has been reported in the literatures [6]. Actually, air gaps act as a non-magnetic phase affecting on the magnetization vector rotation and domain wall displacement. The damping of domain wall displacement is mainly ascribed to both eddy current and hysteresis losses. During the measurement of inductance and resistance of solenoid, the applied alternating magnetic field is weak, thus the contribution of hysteresis effect could be neglected. Furthermore, the hysteresis and eddy current losses are proportional to the first and second power of frequency, respectively. 
Hence with enhancement of frequency, the portion of eddy current loss in the total losses increases. Therefore, the damping of wall displacement and thus, the loss of imaginary part of permeability could be regarded as a result of only eddy current loss [8].

In the plots of imaginary part of permeability there is peak value corresponding to the relaxation frequency (resonant frequency). This point conforms approximately to the value at which the real part of complex permeability begins to be smoothed. This behavior has been also observed for Fe particles coated by phenol-formaldehyde resin (shell) modified with nanometer-sized $\mathrm{SiO}_{2}$ [10].

The interesting point is that when the content of borosilicate is $1 \mathrm{wt} \%$, the real part of permeability of sample with higher density falls to the lower values than that of the sample with lower density with increase of frequency. Although, this drop is insignificant in value, it could be due to the elimination of porosities which enhance the electrical resistivity and act as barriers for flow of eddy currents at high frequencies. However, this fact seems to be not true in the composite with $3 \mathrm{wt} \%$ of borosilicate. In this composite, the effect of higher amount of borosilicate is probably more effective than the elimination of air gaps on the fall of permeability. In addition, the decreasing slop of real part of permeability is more intensive in the sample with higher density.

The relationship between eddy current loss, frequency and electrical resistivity is expressed as follows:

$$
P_{e}=\frac{C B^{2} f^{2} d^{2}}{\rho}
$$

where, B is the magnetic flux density, $\mathrm{f}$ is the frequency, $\mathrm{d}$ is the particles size and $\rho$ is the electrical resistivity [4]. As could be inferred from equation.2, by increasing the frequency and reducing the electrical resistivity at a constant particles size the eddy current loss enhances.

Since the compaction of powders with $3 \mathrm{wt} \%$ of borosilicate was very difficult needing special design of die, the composites with $2 \mathrm{wt} \%$ of borosilicate was selected for the following studies.

\section{3-1-2- The effect of iron particles size in composites with $2 \mathrm{wt} \%$ of borosilicate}

The change of relative density with particles size of iron powders has been illustrated in Fig. 6. As could be observed, the higher density is obtained in the samples with coarser particles size. As it is known, the mechanism of densification at high pressures is the plastic deformation of particles. This mechanism comprises of different steps namely: particles rearrangement, 
localized deformation, homogeneous deformation and bulk densification. The first reaction of powder against pressure is the rearrangement of particles. After that the plastic deformation occurs at the contact points resulting in the work hardening of particles. At the final stages of compaction, the mobile dislocations should be generated to provide the necessary plastic deformation, thus, the densification becomes more difficult and the pressures needed to fill the closed porosities enhance drastically [19]. It could be said that the plastic deformation and consequently, the densification is easier in the coarser particles. When the particles are coarse, the size of pores and porosities is larger than the reverse case with the fine particles. Hence, the elimination of these pores could be accomplished more easily. When the powder particles are under pressure, the shear stresses are created in the regions only adjacent to the pores. In fact, a critical space is defined for generation of dislocations. As the pores are smaller than that critical space the dislocations could not be generated and the densification can't be carried out anymore. In addition, the inter particles friction and bridging effects which are more pronounced in the powders with fine particles could be another reason of achieving lower densities at the same pressures in these powders.

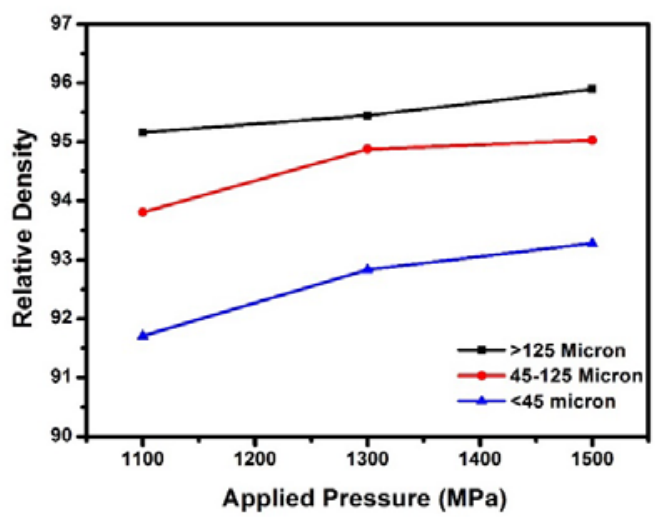

Fig.6. The density as a function of compaction pressure in the samples with different iron particles sizes. The microstructure of samples with different iron particles sizes was investigated via FESEM. Fig. 7 shows the back-scattered and secondary images of samples with iron particles size of $d<45,45<d<125$ and $d>125 \mu \mathrm{m}$. In these images the high density of porosities could be observed which confirms the insufficient flow of borosilicate during sintering. The other point that could be inferred from these images is the size of regions which was filled by the flow of borosilicate. The higher magnification images show that the size of porosities filled by borosilicate is going to be increased when the particles become coarse. The composition of light and dark regions 
measured by EDX has been collected in Table 3 indicating that the mentioned regions are correlated to the iron and borosilicate, respectively.
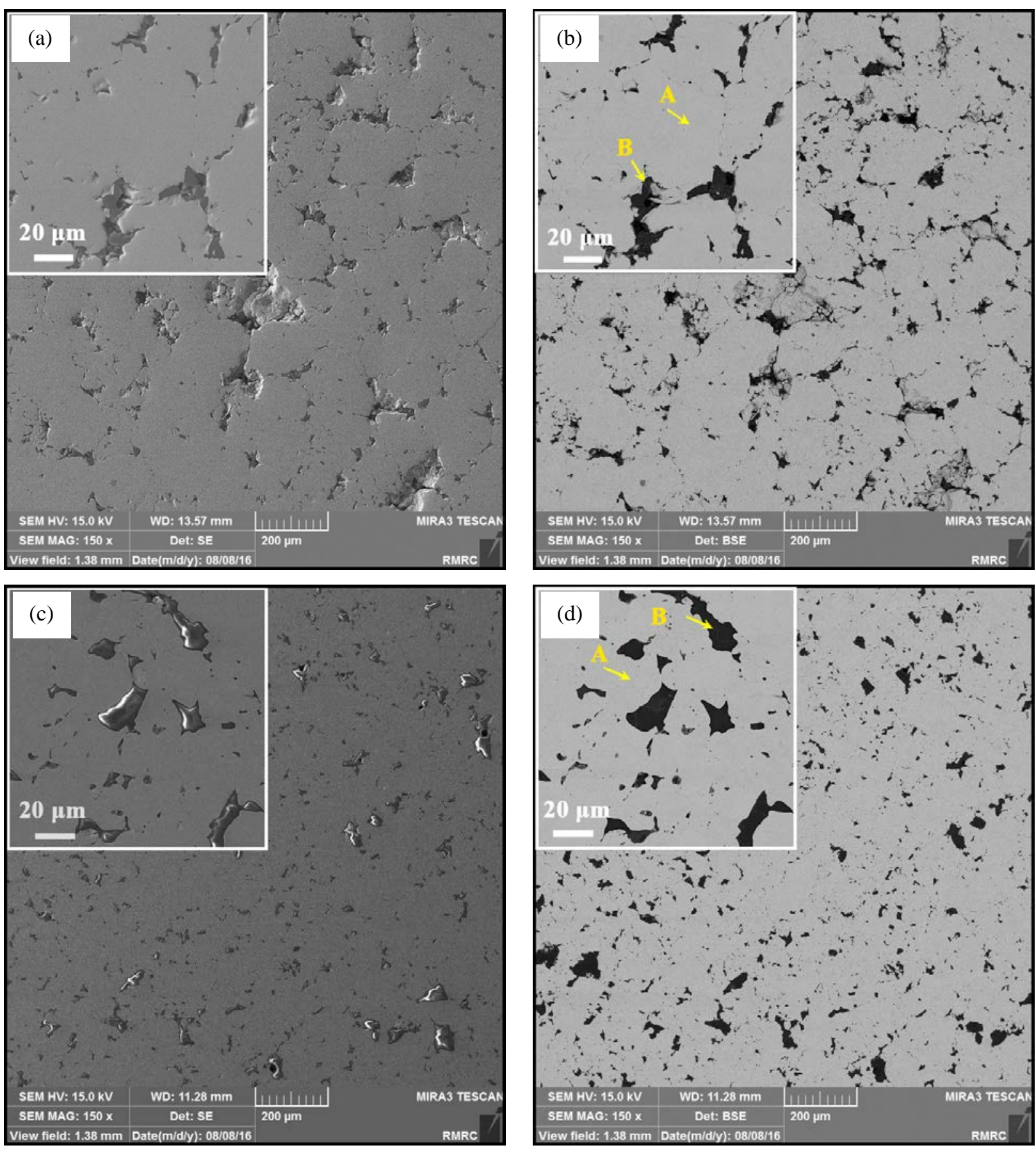

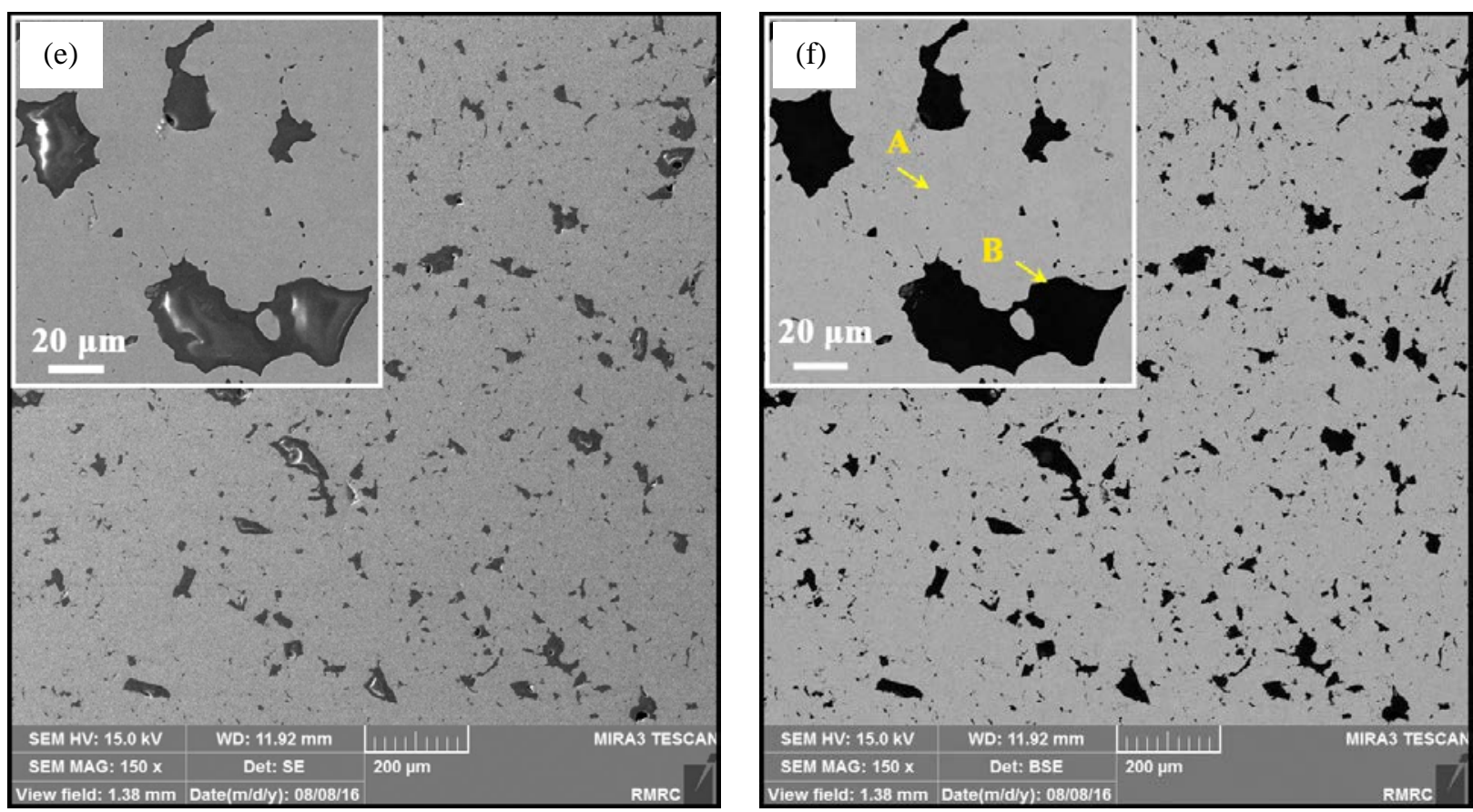

Fig.7. The secondary and back-scattered images of microstructure of composites with initial iron particles size of a,b) d $<45$, c,d) $45<$ d $<125$ and e,f) $d>125 \mu \mathrm{m}$.

Table 3. EDX analysis of different phases shown in the SEM images on Fig.7

\begin{tabular}{c|c|c|c|c|c|c|c}
\hline $\begin{array}{c}\text { The size of iron particles } \\
\text { in composite }(\mu \mathrm{m})\end{array}$ & $\begin{array}{c}\text { Elements } \\
\text { (atomic percent) }\end{array}$ & Fe & B & O & Na & Al & Si \\
\hline \multirow{2}{*}{$\mathrm{d}<45$} & A & 100 & - & - & - & - & - \\
\cline { 2 - 9 } & B & 0.36 & 22.17 & 59.63 & 1.4 & 0.87 & 15.57 \\
\hline \multirow{2}{*}{$45<\mathrm{d}<125$} & A & 100 & - & - & - & - & - \\
\cline { 2 - 9 } & B & 1.18 & 21.91 & 59.80 & 1.46 & 0.71 & 14.95 \\
\hline \multirow{2}{*}{$\mathrm{d}>125$} & A & 100 & - & - & - & - & - \\
\cline { 2 - 8 } & B & 1.51 & 21.37 & 60.16 & 1.53 & 0.71 & 14.71 \\
\hline
\end{tabular}

The influence of iron particles size on the electrical resistivity has been also investigated. The obtained resistances were close to each other in samples with different particles sizes in each 
compaction pressure. Only very low increment was observed with increasing particles size. The resistivity increased from 13 to $15.5 \mu \Omega$.m when the particles size of samples increased from $\mathrm{d}<45$ to $\mathrm{d}>125 \mu \mathrm{m}$. This insignificant and negligible enhancement could be due to the existence of larger porosities in the samples with larger particles size before sintering which could be filled by the flow of borosilicate at its softening temperature during sintering enhancing the electrical resistivity. These pores have been shown in the FESEM images. Furthermore, the amount of the open porosities before sintering calculated by means of Archimedean method has been listed in Table. 4.

Table 4. The percent of open porosities in the samples with different particles sizes

\begin{tabular}{c|c}
\hline Iron particle size $(\mu \mathrm{m})$ & The percent of open porosities \\
\hline $\mathrm{d}<45$ & 2.67 \\
\hline $45<\mathrm{d}<125$ & 2.77 \\
\hline $\mathrm{d}>125$ & 2.92 \\
\hline
\end{tabular}

Fig. 8 depicts the real and imaginary parts of complex permeability for samples with 2 wt $\%$ of borosilicate at different iron particles sizes. The trend of change of permeability is similar to that observed in the previous section. As could be seen the value of real part of permeability is higher in the sample with coarser iron particles size. As the particles size decreases the number of particles at the same amount of magnetic material increases compared to the large particles. Thus the surface to volume ratio and consequently, the amount of particles walls and defects increases as well. Accordingly, the gaps between particles raise leading to the enhancement of demagnetizing field which retards the magnetization process, as a consequence the permeability decreases [20].

The other point that could be deduced from the imaginary part of permeability is the decrease of relaxation frequency with increase of particles size. Increasing the particles size from $\mathrm{d}<45$ to $\mathrm{d}>125 \mu \mathrm{m}$ led to the decrease of relaxation frequency from 480 to $420 \mathrm{~Hz}$.

The relationship between relaxation frequency and particles size is defined as follows:

$$
f_{r}=\frac{\rho}{2 \pi \mu_{0} \mu_{i} d^{2}}
$$

where, $\rho$ is specific electrical resistivity, $d$ is particles size, $\mu_{\mathrm{i}}$ is the initial permeability and $\mu_{0}$ is the permeability of the vacuum [13]. According to this equation, the increase of particles size yields to the decrease of relaxation frequency. 

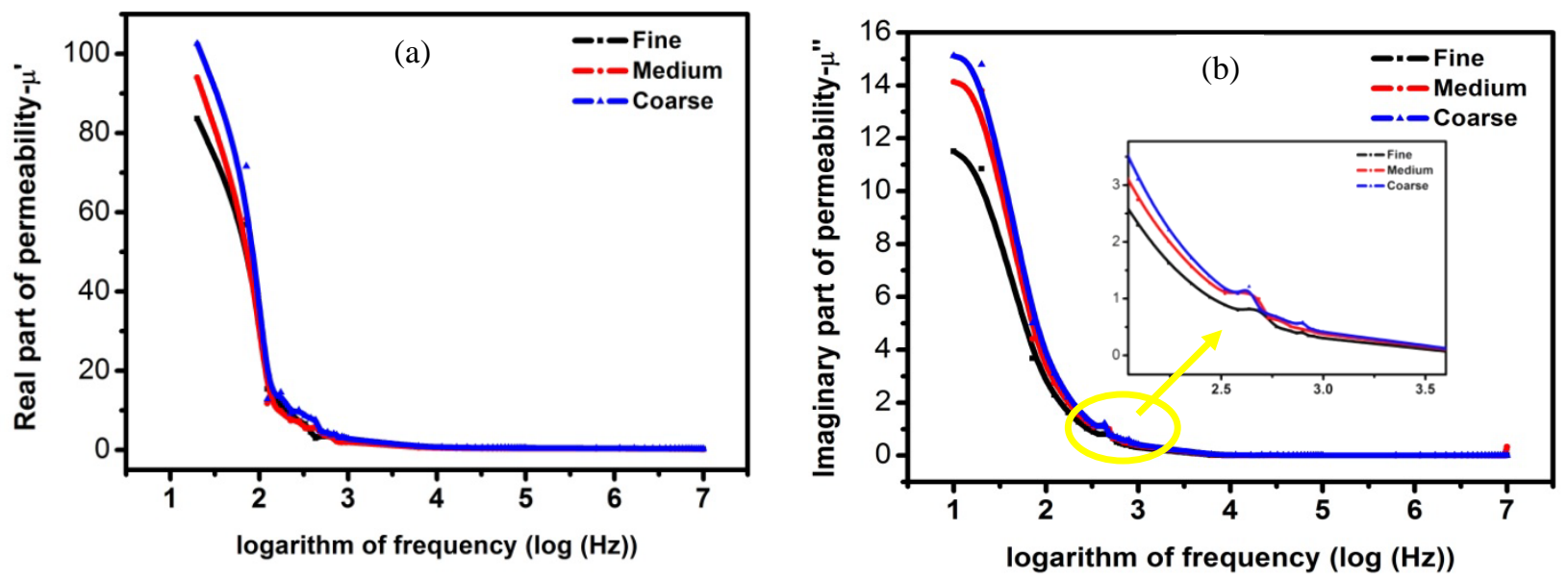

Fig.8. a) Real and b) imaginary parts of complex permeability for composites containing $2 \mathrm{wt} \%$ of borosilicate with different particles sizes.

The hysteresis loop for sample with highest density (consolidated with pressure of $1500 \mathrm{MPa}$ ) and particles size larger than $125 \mu \mathrm{m}$ has been exhibited in Fig.9. The hysteresis loop is similar to the characteristic shape for soft magnetic materials. The magnetization and coercivity of this sample have been obtained as $180 \mathrm{emu} / \mathrm{g}$ and 7 Oe, respectively.

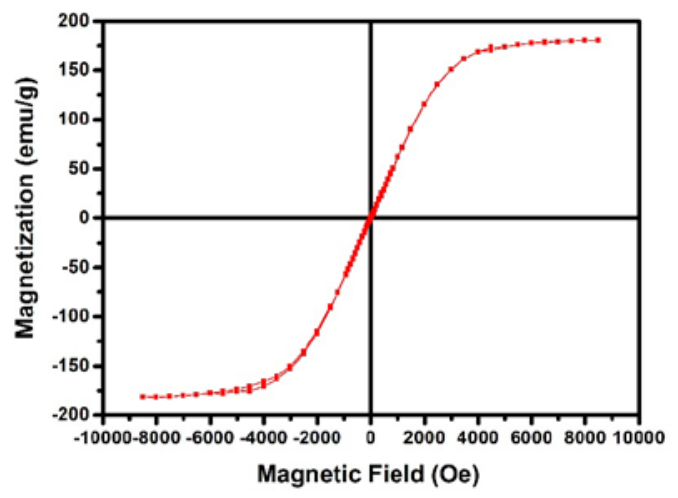

Fig.9. The DC hysteresis loop of sample with $2 \mathrm{wt} \%$ of borosilicate and iron particles size $\mathrm{d}>125 \mu \mathrm{m}$.

\section{3-2- The compaction by spark plasma sintering}

Since the samples consisting of $2 \mathrm{wt} \%$ of borosilicate and having coarse particles reach to the higher density (Fig.1 and Fig.6) and electrical resistivity rather than the other samples during 
compaction by uniaxial pressure, the powders with particles size of $45<\mathrm{d}<125$ and $\mathrm{d}>125 \mu \mathrm{m}$ were chosen for spark plasma sintering.

The density and electrical resistivity of mentioned composites have been collected in Table 5. As could be inferred from this Table, the higher density has been obtained in spark plasma sintered samples than those consolidated by uniaxial compaction technique. This confirms the achievement of near full dense samples by spark plasma sintering process.

Table 5. The density and electrical resistivity of spark plasma sintered samples

\begin{tabular}{c|c|c|c}
\hline Iron particle size $(\mu \mathrm{m})$ & Density & Relative density $(\%)$ & Electrical resistivity $(\mu \Omega . \mathrm{m})$ \\
\hline $45<\mathrm{d}<125$ & 7.412 & 99.90 & 6.8 \\
\hline $\mathrm{d}>125$ & 7.405 & 99.80 & 5.3 \\
\hline
\end{tabular}

In addition; the electrical resistivity of these two samples are close to each other but lower than those obtained by uniaxial pressing. Indeed, the high density achieved in this sample causes the high connectivity between particles leading to the decrease of electrical resistivity.

The microstructure of these samples has been studied by FESEM. As could be seen in Fig.10, the better distribution of borosilicate has been obtained by applying the spark plasma sintering technique. Similar to the previous results and based on the EDX data listed in Table 6, the dark regions marked by letter B are ascribed to the borosilicate phase. In the secondary images any porosity could not be seen verifying the fabrication of near full dense composite. 

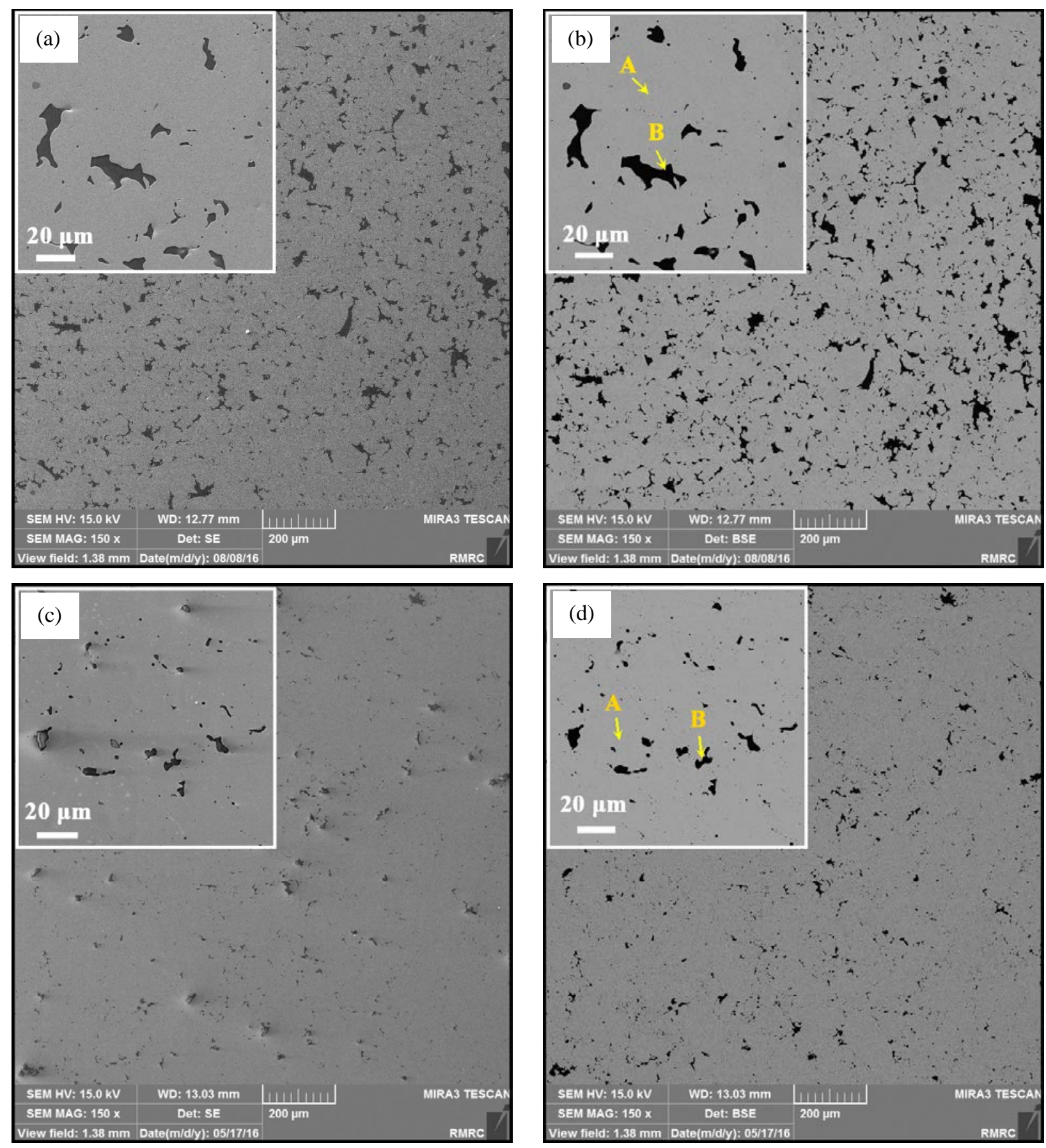

Fig.10. The secondary and back-scattered images of microstructure of composites made by spark plasma sintering with initial iron particles size of a,b) $45<\mathrm{d}<125$ and c,d) $d>125 \mu \mathrm{m}$. 
Table 6. EDX analysis of different phases shown in the SEM images on Fig.7

\begin{tabular}{c|c|c|c|c|c|c|c}
\hline $\begin{array}{c}\text { The size of iron particles } \\
\text { in Composite }(\mu \mathrm{m})\end{array}$ & $\begin{array}{c}\text { Elements } \\
\text { (atomic percent) }\end{array}$ & Fe & B & O & Na & Al & Si \\
\hline \multirow{2}{*}{$45<\mathrm{d}<125$} & A & 100 & - & - & - & - & - \\
\cline { 2 - 8 } & B & 4.16 & 19.1 & 59.84 & 1.61 & 1.03 & 14.26 \\
\hline \multirow{2}{*}{$\mathrm{d}>125$} & A & 100 & - & - & - & - & - \\
\cline { 2 - 8 } & B & 2.38 & 17.46 & 61.5 & 1.94 & 0.88 & 15.84 \\
\hline
\end{tabular}

Fig. 11 indicates the real and imaginary parts of complex permeability in the composites made by spark plasma sintering with different iron particles sizes. It is apparent that both real and imaginary values are too close to each other in these two composites. Furthermore, the values of permeability are higher than those fabricated by uniaxial pressing. As an example, the maximum real part of permeability in the composite with iron particles size larger than $125 \mu \mathrm{m}$ is 106 which is higher than $\mu^{\prime}=101$ produced by uniaxial pressing. Similar to the previous results, the relaxation frequency is a bit smaller in the sample with larger particles size.
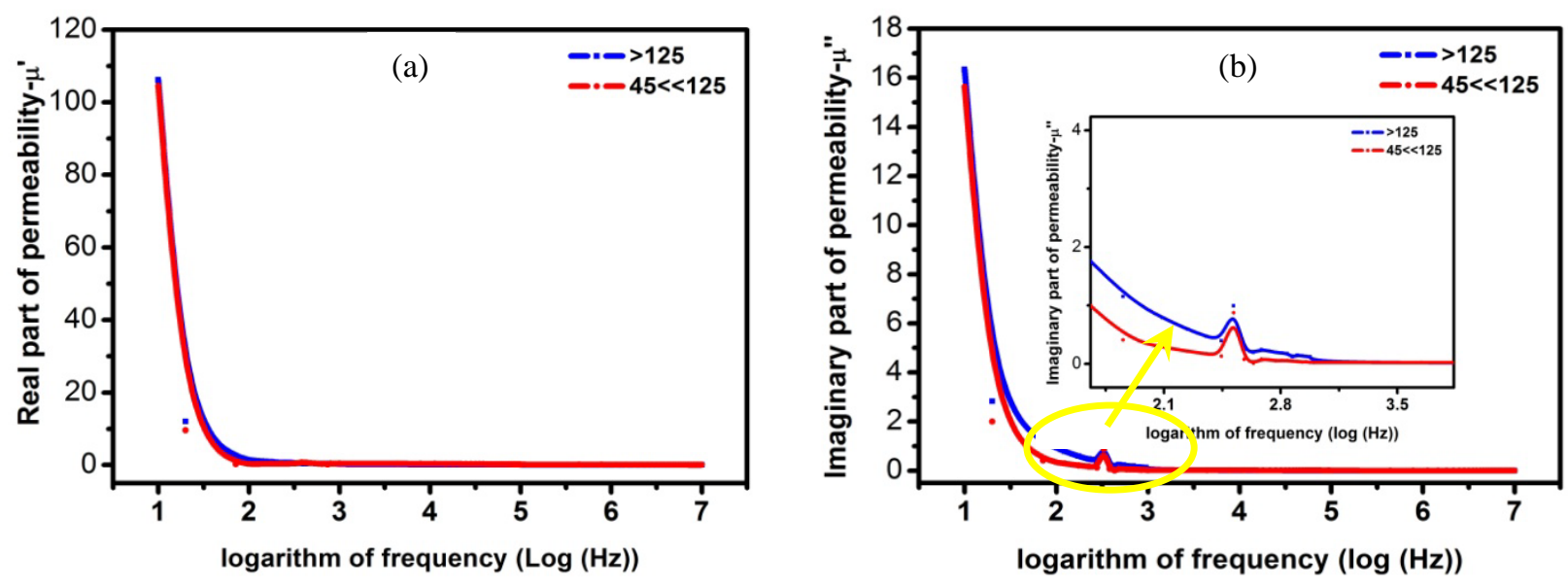

Fig.11. a) Real and b) imaginary parts of complex permeability for composites made by spark plasma sintering with borosilicate content of $2 \mathrm{wt} \%$ and different initial particles sizes.

Fig.12 shows the hysteresis loop for samples made by spark plasma sintering. The achievement of near full density by spark plasma sintering yields to the high magnetization and low demagnetization fields. The magnetization of both composites made by this technique has been 
obtained 180 emu/gr. Moreover, the coercivity has been attained as 33 and 20 Oe for composites with particles size of $45<\mathrm{d}<125$ and $\mathrm{d}>125 \mu \mathrm{m}$, respectively. These values are comparable with those given in the other researches $[5,10]$. Here, the higher values obtained for coercivity may be owing to the shorter heating times, which leads to the less stress relieving.

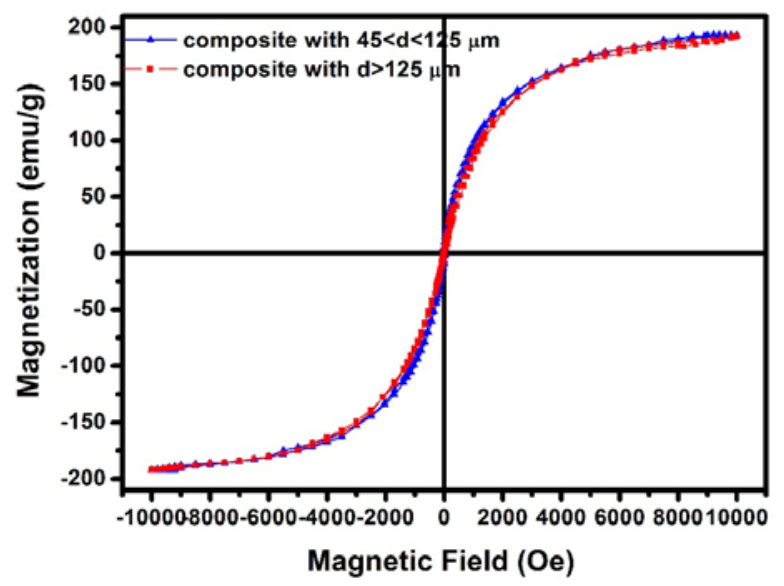

Fig.12. The DC hysteresis loop of the samples with 2 wt\% of borosilicate made by spark plasma sintering.

\section{Conclusion}

In this work, iron- borosilicate soft magnetic composites have been produced by two different techniques: uniaxial pressing following by sintering and spark plasma sintering. It was shown that borosilicate is distributed on the grain boundaries of iron causing the increase of resistivity and decrease of eddy currents. By enhancement of compaction pressure the air gaps between particles decreased and consequently, the density increased leading to decrease of electrical resistivity. Increasing the frequency resulted in the decrease of both real and imaginary parts of permeability. This drop is steep at the initial frequencies, but then it becomes smooth. The drop is also less intensive in the composite with higher content of borosilicate. Best combination of density, electrical resistivity and magnetic properties (at AC and DC applications) including permeability, magnetization and coercivity was obtained in the composite with 2 wt\% of borosilicate. Moreover, by increasing the iron particles size the density increased. In addition, the electrical resistivity showed an insignificant increase which could be due to the larger size of open porosities before sintering where the borosilicate could aggregate. It could also be owing to the more inter-particles friction and more bridging effects in powders with finer particles size. The permeability of composites with fine particles size is lower than that of coarse grains due to the demagnetizing fields arising from the high amounts of surface monopoles which are more 
pronounced in finer particles. Furthermore, enhancing the particles size from $\mathrm{d}<45$ to $\mathrm{d}>125 \mu \mathrm{m}$ resulted in the decrease of relaxation frequency from 480 to $420 \mathrm{~Hz}$. Near full density (99.99 \% of theoretical density) with homogenous distribution of borosilicate was achieved in the composites made by SPS. The higher density of samples made by SPS than those fabricated by uniaxial pressing led to the greater amounts of real and imaginary parts of permeability.

\section{Acknowledgement}

This research was financially supported by Iran National Science Foundation (The Office of President, Vice-Presidency for Science and Technology) under the grant number of 93032200.

\section{References}

[1] Y.G. Guo, J.G. Zhu, Z.W. Lin, J.J. Zhong, 3D vector magnetic properties of soft magnetic composite material, Journal of Magnetism and Magnetic Materials, 302 (2006) 511-516.

[2] Y.G. Guo, J.G. Zhu, Applications of soft magnetic composite materials in electrical machines, Australian Journal of Electrical and Electronics Engineering, 3 (2006) 37-46.

[3] I. Hemmati, H.R. Madaah Hosseini, A. Kianvash, The correlations between processing parameters and magnetic properties of an iron-resin soft magnetic composite, Journal of Magnetism and Magnetic Materials, 305 (2006) 147-151.

[4] H. Shokrollahi, K. Janghorban, Soft magnetic composite materials (SMCs), Journal of Materials Processing Technology, 189 (2007) 1-12.

[5] J. Füzer, J. Füzerová, P. Kollár, M. Fáberová, R. Bures, iron based soft magnetic compacted materials, Acta Electrotechnica et Informatica, 13 (2013) 82-85.

[6] A.H. Taghvaei, H. Shokrollahi, M. Ghaffari, K. Janghorban, Influence of particle size and compaction pressure on the magnetic properties of iron-phenolic soft magnetic composites, Journal of Physics and Chemistry of Solids, 71 (2010) 7-11.

[7] M.M. Dias, H.J. Mozetic, J.S. Barboza, R.M. Martins, L. Pelegrini, L. Schaeffer, Influence of resin type and content on electrical and magnetic properties of soft magnetic composites (SMCs), Powder Technology, 237 (2013) 213-220.

[8] J. Fuzerova, J. Fuzer, P. Kollar, L. Hegedus, R. Bures, M. Faberova, Analysis of the Complex Permeability Versus Frequency of Soft Magnetic Composites Consisting of Iron and Fe73.5Cu1 Nb3Si16B7, IEEE Transactions on Magnetics, 48 (2012) 1545-1548.

[9] S. Wu, A. Sun, F. Zhai, J. Wang, Q. Zhang, W. Xu, P. Logan, A.A. Volinsky, Annealing effects on magnetic properties of silicone-coated iron-based soft magnetic composites, Journal of Magnetism and Magnetic Materials, 324 (2012) 818-822.

[10] M. StreČKovÁ, J. FÜZer, L. MedveckÝ, R. BureŠ, P. KollÁR, M. FÁBerovÁ, V. Girman, Characterization of composite materials based on Fe powder (core) and phenol-formaldehyde resin (shell) modified with nanometer-sized SiO2, Bulletin of Materials Science, 37 (2014) 167177.

[11] M. Yaghtin, A.H. Taghvaei, B. Hashemi, K. Janghorban, Effect of heat treatment on magnetic properties of iron-based soft magnetic composites with Al2O3 insulation coating produced by sol-gel method, Journal of Alloys and Compounds, 581 (2013) 293-297. 
[12] S. Wu, A. Sun, Z. Lu, C. Cheng, Fabrication and properties of iron-based soft magnetic composites coated with parylene via chemical vapor deposition polymerization, Materials Chemistry and Physics, 153 (2015) 359-364.

[13] S. Wu, A. Sun, W. Xu, Q. Zhang, F. Zhai, P. Logan, A.A. Volinsky, Iron-based soft magnetic composites with Mn-Zn ferrite nanoparticles coating obtained by sol-gel method, Journal of Magnetism and Magnetic Materials, 324 (2012) 3899-3905.

[14] K. Gheisari, S. Javadpour, H. Shokrollahi, B. Hashemi, Magnetic losses of the soft magnetic composites consisting of iron and Ni-Zn ferrite, Journal of Magnetism and Magnetic Materials, 320 (2008) 1544-1548.

[15] M. Wang, Z. Zan, N. Deng, Z. Zhao, Preparation of pure iron/Ni-Zn ferrite high strength soft magnetic composite by spark plasma sintering, Journal of Magnetism and Magnetic Materials, 361 (2014) 166-169.

[16] Y. Peng, J. Nie, W. Zhang, C. Bao, J. Ma, Y. Cao, Preparation of soft magnetic composites for Fe particles coated with (NiZn)Fe2O4 via microwave treatment, Journal of Magnetism and Magnetic Materials, 395 (2015) 245-250.

[17] S. Wu, A. Sun, Z. Lu, C. Cheng, X. Gao, Magnetic properties of iron-based soft magnetic composites with $\mathrm{SiO} 2$ coating obtained by reverse microemulsion method, Journal of Magnetism and Magnetic Materials, 381 (2015) 451-456.

[18] T. Gheiratmand, H.R. Madaah Hosseini, P. Davami, C. Sarafidis, Fabrication of FINEMET bulk alloy from amorphous powders by spark plasma sintering, Powder Technology, 289 (2016) 163-168.

[19] P.W. Lee, Y. Trudel, R. Iacocca, R.M. German, B.L. Ferguson, W.B. Eisen, K. Moyer, D. Madan, H. Sanderow, ASM Metals Handbook, powder metal technologies and applications, volume 7, 1998.

[20] M. Anhalt, Systematic investigation of particle size dependence of magnetic properties in soft magnetic composites, Journal of Magnetism and Magnetic Materials, 320 (2008) e366-e369. 